\title{
mRNP quality control goes regulatory
}

\author{
Oliver Mühlemann ${ }^{1}$ \& Torben Heick Jensen ${ }^{2}$ \\ ${ }^{1}$ Dept. of Chemistry and Biochemistry, University of Bern, Freiestrasse 3, $\mathrm{CH}$ - \\ 3012 Bern, Switzerland \\ ${ }^{2}$ Centre for mRNP Biogenesis and Metabolism, Dept. of Molecular Biology \\ and Genetics, C.F. Møllers Allé 3, Building 1130, Aarhus University, 8000 \\ Aarhus C., Denmark
}

Corresponding authors:

Mühlemann, O. (oliver.muehlemann@dcb.unibe.ch)

Jensen, T.H. (thj@mb.au.dk) 


\section{Abstract}

The accuracy of eukaryotic gene expression relies on efficient quality control (QC). Virtually all steps in the gene expression pathway en route from transcription to translation are error-prone and QC systems have evolved to utilize many of these biochemical processes as checkpoints to monitor the production or function of mRNA-protein particles (mRNPs). Mechanistically, such evaluation of mRNP fitness is based on a competition between the opposing activities of mRNP biogenesis/function and turnover. In fact, quite subtle alteration of any parameter can tip the balance between mRNP persistence and degradation, and therefore QC checkpoints also comprise perfect opportunities for controlling cellular levels of individual or even entire families of transcripts. From this perspective, QC and gene regulation represent two outcomes of the same molecular process. 


\section{Omnipresent mRNP QC}

The life of a eukaryotic mRNA is astonishingly complex: from its birth in the nucleus to its cytoplasmic demise it undergoes a series of interconnected biochemical reactions, starting with its synthesis by RNA polymerase II (RNAPII), followed by the transcription-coupled addition of a 7-methylguanylate cap to its 5' end, removal of its introns and cleavage and polyadenylation of its $3^{\prime}$ end ${ }^{1-3}$. In addition to these covalent chemical changes, each mRNA has to correctly interact with a specific ensemble of RNA-binding proteins to form the mRNP that constitutes the functional entity of the message. Some of these proteins accompany the mRNA all the way until it is translated or degraded, while others associate in a location-specific or temporal manner to convey properties to the mRNP of only transient utility. Regardless of their exact nature, steps in the biogenesis and remodelling of mRNPs are susceptible to mistakes. To reduce the error frequency of the steady-state mRNP population, cells have evolved QC systems at several levels in both the nucleus and in the cytoplasm ${ }^{4-7}$ (Figure 1). These often take advantage of the normal reactions of mRNP biogenesis or utility where the welfare of the concerned mRNP is directly revealed. Indeed, most QC systems described so far intervene if the efficiency by which the mRNP is produced and transported is inadequate or if it performs poorly in translation. Current examples of QC checkpoints include the processes of mRNA capping ${ }^{8,9}$, splicing ${ }^{10}, 3^{\prime}$-end formation ${ }^{11-15}$, mRNP nuclear exit ${ }^{16-18}$ and interaction with ribosomes ${ }^{19-25}$.

For an in-depth discussion of the constituent factors of QC systems and their mechanisms of action, we refer the reader to recent reviews on nuclear ${ }^{5-}$ 
7 and cytoplasmic QC ${ }^{26-30}$. Here, we emphasize that QC systems are not dedicated entities evolved only to survey mRNP performance. Instead, QC is often based on the somewhat surprising intimate connection between common cellular RNA degradation activities and factors involved in productive mRNP transactions. In fact, degradation factors, or the proteins recruiting them, often participate themselves in mRNP production or remodelling. The term QC therefore often describes a competition between the opposing processes of biogenesis/function and turnover, which permits the elimination of molecules or complexes that do not meet certain standards. Interestingly however, although QC processes normally serve to remove aberrant material, they can be readily exploited by the cell to also serve gene regulatory purposes, and because most mRNP biogenic and metabolic activities are functionally intertwined ${ }^{2,3}$, there is ample opportunity for such regulation. Here, we aim to illustrate this concept by recently published examples.

\section{Balancing biogenesis and function with decay}

Whenever an mRNP fails to pass QC, its progression is restricted with the typical result that its mRNA component is degraded by ribonucleases. Recycling of particles retained by QC systems back into the functional pool is a formal possibility, but published examples are lacking. Likewise, repair of defective RNA can occur in special cases as part of stress response pathways ${ }^{31}$, but instances of aberrant mRNA repair by $Q C$ systems have so far not been reported. Instead, and as mentioned above, the kinetic competition between steps in normal mRNP biogenesis or function and RNA turnover represents a unifying principle in many QC processes (reviewed in ${ }^{4}$ 
6, 32). The outcome of this competition between normal maturation/function and commitment to mRNA degradation can be viewed as a double pan balance in which one pan contains mRNPs that passed QC and the other contains complexes whose mRNAs are destined for degradation (Figure 2).

For any individual mRNP species, the fraction ending up in either pan of the balance ultimately depends on the competing rate constants, which we refer to as $k_{\text {normal }}$ (rate constant for the normal maturation step or function) and $k_{\text {aberrant }}$ (rate constant for commitment to mRNA decay). Although QC systems are not yet understood in sufficient detail to allow a precise determination of these rate constants and make quantitative statements, they are still very useful as tools for discussion. Due to the inherent error rate of every step of mRNA production and mRNP assembly, any population of a specific mRNP species will harbour a fraction of particles that are defective or have reduced functionality. These mRNPs have a lower $k_{\text {normal }} / k_{\text {aberrant }}$ ratio compared to their flawless mRNP cousins, resulting in their differential distribution between the two pans and hence in efficient QC (Figure 2A). For example, aberrant mRNPs may be less efficient substrates for the processing reaction or functional activity in question (i.e. a reduced $k_{\text {normal }}$ ) or they may have acquired degradation-promoting features (i.e. an increased $k_{\text {aberrant }}$ ). Notably, when the overall capacity of a particular decay pathway is exceeded because of suboptimal mRNP production/function and/or because a key degradation factor is inactivated, aberrant mRNAs will accumulate in the cell. Indeed, it is under such conditions that most QC pathways have been revealed experimentally. 
If we apply the double pan balance concept to the entire cellular population of particles, each mRNP species will have its own intrinsic average $k_{\text {normal }} / k_{\text {aberrant }}$ ratio that determines which fraction will survive a given $Q C$ checkpoint. Thus, extrinsically induced changes affecting the overall assembly, processing or degradation rates, e.g. by modification of the activity of an enzyme required for mRNP production/function or decay, will alter the species-specific $k_{\text {normal }} / k_{\text {aberrant }}$ ratio and hence the proportion of surviving mRNPs (Figure 2B). This is because mRNA-inherent features influencing $k_{\text {normal }}$ or $k_{\text {aberrant }}$ are diverse and of different strengths, hereby providing a wide and gradual spectrum of possible responses to e.g. stress or changed growth conditions. The concentration of a single mRNP species may also be specifically regulated via alteration of one or several of its constituents, changing the $k_{\text {normal }}$ and/or $k_{\text {aberrant }}$ of that species only. In such considerations, the system controls gene expression and the distinction between mRNP QC and gene regulation becomes arbitrary.

\section{Nuclear QC systems at work}

As a general nuclear surveillance measure, QC systems in this locale efficiently remove mRNAs undergoing slow or aberrant 5' capping, splicing or 3'-end formation (reviewed in ${ }^{6,}{ }^{33}$ ). Moreover, QC deals with malformed mRNPs, presumably via their inability to mature in a timely manner ${ }^{14}$. While it is not known how much 'QC activity' is invested in these tasks during normal cellular growth, studies from several eukaryotic model systems have shown that nuclear QC machineries are constantly kept busy by the ubiquitous activity of RNAPII outside protein-coding genes. This is because large 
portions of eukaryotic genomes are transcribed, often on both strands and in a quite promiscuous manner ${ }^{34-36}$. As many of these transcripts are not destined for cytoplasmic translation or immediate nuclear usage ${ }^{37-41}$, the majority are under physiological conditions rapidly removed and accumulate only when QC components (e.g. the 3'-5' exo- and endoribonucleolytic RNA exosome or its co-factors) are artificially inhibited 37,41 . Hence, cryptic unstable transcripts (CUTs) from Saccharomyces cerevisiae ${ }^{37}$ or promoter upstream transcripts (PROMPTs) from human cells ${ }^{41,42}$ constitute examples where the balance between maturation and decay is tilted virtually all the way towards complete degradation. In S. cerevisiae, transcription termination of CUTs by the Nrd1p/Nab3p/Sen1p complex is aided by Nrd1p/Nab3p binding sites in the affected RNA and is directly coupled to their degradation by the RNA exosome aided by its co-activator TRAMP ${ }^{39}$. Although human exosome co-factors involved in PROMPT turnover have recently been identified, it is not yet clear how tightly the degradation of these transcripts is linked to the transcription termination event ${ }^{43}$. Finally, whether situations exist that tip the balance towards preferential stabilization of CUTs/PROMPTs to yield potentially functional RNAs remains to be seen.

\section{Shifting the balance: Selective recruitment of QC activity}

CUT transcription can provide regulatory function by impacting the activity of a closely positioned downstream gene ${ }^{44-47}$. This regulation is imposed by the CUT transcription event itself and therefore likely leaves the resulting CUT RNA as a disposable by-product for exosomal degradation. In related instances, the Nrd1p/Nab3p/Sen1p complex is also involved in the premature 
transcription termination of genes, hereby hindering their production of fulllength gene transcripts. The NRD1 gene itself is a particularly interesting case as it is subjected to auto-regulation by Nrd1p-dependent termination and exosomal degradation in about $80 \%$ of all transcription events ${ }^{48}$ (Figure $3 \mathrm{~A}$ ). Such dramatic dampening of NRD1 mRNA synthesis is guided by the presence of Nrd1p/Nab3p binding sites in the 5' UTR and coding region of the NRD1 mRNA. Thus, gene expression can be selectively downregulated by directed recruitment of $\mathrm{QC}$ factors. An intriguing glimpse of potentially complex interactions impacting this regulation was recently provided by the demonstration that the $S$. cerevisiae MAP kinase Mpk1p can prevent Nrd1p/Nab3p/Sen1p-dependent transcription termination of the FKS2 gene by blocking the recruitment of the complex to RNAPII ${ }^{49}$.

Exploitation of QC factors for gene regulatory purposes also occurs at the post-transcriptional level. In one example, the auto-regulated expression of the NAB2 gene, encoding the $S$. cerevisiae nuclear RNA poly $(\mathrm{A})$ binding protein (PABP), relies on a stretch of 26 adenosine residues in the NAB2 $3^{\prime}$ UTR ${ }^{50}$ (Figure 3B). Proper 3' end formation of NAB2 mRNA appears to occur by the non-conventional 3' trimming of extended NAB2 mRNA species by the nuclear exosome component Rrp6p, and the TRAMP factor Trf4p, eventually resulting in polyadenylation near or within the $A_{26}$ stretch ${ }^{51}$. Presumably via binding to the $A_{26}$ sequence, rising levels of Nab2p prevents polyadenylation at this site and instead shifts the balance towards Rrp6pdependent degradation of NAB2 mRNA. The mechanism by which Nab2p sensitizes the transcript to exosomal-decay is not known, but may depend on the ability of Nab2p to recruit Rrp6p (THJ, unpublished observation). 
Moreover, this involvement of QC factors in the 'normal' 3' end formation process appears to be of a broader nature as a related phenomenon has been reported for the $S$. cerevisae CTH2 mRNA ${ }^{52}$. It is also a strategy utilized in a second, conceptually related example from Schizosaccharomyces pombe. Here, vegetative cells are maintained by the direct recruitment of nuclear RNA exosome activity for the selective elimination of an estimated $>20$ meiotic mRNAs, whose protein products would otherwise adversely affect growth ${ }^{53-56}$ (Figure $3 C$ ). Again, PABP is involved in the process: the nuclear S. pombe Pab2p protein, the homolog of mammalian PABPN1, physically associates with Rrp6p ${ }^{57}$. Remarkably, poly(A) tail addition by the conventional S. pombe poly(A) polymerase, Pla1p, appears to be underlying Pab2p/exosome recruitment to RNA, demonstrating that destabilization through polyadenylation, which is a hallmark for TRAMP-associated enzymes, can also be achieved by a regular poly(A) polymerase ${ }^{56}$. Pab2p/exosomespecificity towards meiosis-specific RNAs is achieved with the help of the RNA-binding protein Mmi1p, which belongs to the $\mathrm{YTH}$ family and interacts with 'determinant of selective removal' (DRS) regions residing in the 3' ends of these transcripts. From this position, Mmi1p is thought to aid Pab2p/Rrp6p recruitment and at the same time interfere with 3 ' end processing of these pre-mRNAs, which as a consequence become easy preys for the exosome ${ }^{54}$. During meiosis, continuous degradation is inhibited, presumably by sequestration of Mmi1p away from meiosis-specific mRNAs and into nuclear 'Mei2 dots', microscopically detectable foci consisting of the meiosis-specific Mei2p protein and additional components ${ }^{53}$. Collectively, these examples of QC factor-mediated post-transcriptional gene regulation challenge the 
traditional view that nuclear PABPs solely function in poly $(A)$ tail biogenesis of regular mRNA: they may in addition facilitate the recruitment of QC factors, resulting in mRNA decay unless the targeted mRNP has evolved other means to deflect this nuclear ribonucleolytic challenge (e.g. preventive cis-elements or trans-factors, or a rapid expedition into the cytoplasm) ${ }^{58}$.

\section{Shifting the balance: Modification of QC activity}

Gene expression control by altering nuclear QC activity has been well documented in the yeasts $S$. cerevisiae and $S$. pombe. It is based on the widespread antisense (as) transcription of these genomes and the ability of RNA degradation activities to rapidly alter cellular levels of such molecules ${ }^{59-}$ ${ }^{64}$. A prime example is the as-transcription through the S. cerevisiae PHO84 gene ${ }^{59,60}$ (Figure 4). Because PHO84 asRNA negatively regulates gene sense transcription through chromatin modification and histone deacetylation of the PHO84 promoter, and because it is a target of the nuclear exosome, QC activity dictates cellular levels of both as and sense RNAs. Along these lines, PHO84 gene silencing is observed in ageing cells, where less Rrp6p is recruited to the PHO84 locus than in young cells ${ }^{60}$. Notably, Rrp6p abundance remains unchanged during ageing, which argues for a posttranslational regulation of the Rrp6p activity, but the exact mechanism still needs to be worked out.

Changing intracellular Rrp6p levels represents an alternative way to modulate Rrp6p activity, as exemplified by the switch from mitosis to meiosis in S. cerevisiae: at the onset of meiosis, Rrp6p is degraded, leading to the stabilization of multiple so-called meiotic unannotated transcripts (MUTs) ${ }^{65}$. 
As diploid cells lacking Rrp6p cannot undergo efficient meiotic development, this sudden switch in the nuclear repertoire of ncRNA may have important consequences for the meiotic gene expression program.

A related example is provided by the asRNA-mediated regulation of $S$. cerevisiae GAL genes required for galactose metabolism ${ }^{61}$. Under conditions of GAL gene repression, asRNA expression through the GAL1-10 cluster leads to recruitment of the $\mathrm{Rpd} 3$ histone deacetylation complex and suppression of the GAL1-10 promoter. The asRNA is also here targeted by TRAMP/exosome, and since exosome activity is affected by carbon source supply ${ }^{10}$, it is tempting to speculate that this constitutes at least part of the GAL gene regulatory circuit. As ncRNAs are thought to participate in the regulation of numerous catabolic genes, modulation of TRAMP/exosome activity is likely to broadly contribute to changes in gene expression programs upon changes in nutrient availability. Moreover, the perspectives for gene expression regulation via control of asRNA levels by the regulation of $\mathrm{QC}$ factor activity are enormous, as turnover of asRNAs is not confined to the exosome. For example, of 1658 Xrn1p-sensitive unstable transcripts (XUTs; ncRNAs destabilized by the major cytoplasmic 5'-3' exonuclease Xrn1p) identified in a recent genome-wide survey, $66 \%$ were antisense to open reading frames ${ }^{62}$. Given the increasing realization that sense-antisense RNA pairs also impact biological processes in higher eukaryotic cells, which also harbour their stocks of yeast-like QC factors, this type of regulation is likely evolutionary conserved.

QC at the level of translation: NMD, NSD and NGD 
Even after mRNPs engage with the translation apparatus, QC ensues.

Failure of ribosomes to terminate translation properly commonly triggers the rapid destruction of the concerned mRNA 1, 4, 26, which under certain conditions is accompanied by degradation of the involved ribosomes ${ }^{66,67}$. Well documented examples include transcripts harboring a premature termination codon (PTC) targeted by nonsense-mediated decay (NMD) (reviewed in ${ }^{30} 27,29$ ), mRNAs that lack a termination codon targeted by nonstop mRNA decay (NSD) ${ }^{19,20}$, and mRNAs with ribosomes stalled at stable secondary structures or at a stretch of rare codons targeted by no-go mRNA decay (NGD) ${ }^{21}$.

Recent studies suggest that NSD and NGD might be mechanistically related processes. First, translation of the poly $(\mathrm{A})$ tail of a nonstop mRNA generates a poly-lysine chain that was reported to stall ribosomes by clogging their exit tunnels ${ }^{68}$, which essentially creates a no-go situation. Second, NSD and NGD both involve the release factor eRF1 homolog Pelota (Dom34p in yeast) and the eRF3 homologous GTPase Hbs1p ${ }^{21,69}$. In addition to these two release factor-mimicking proteins ${ }^{70,71,72}$, the yeast E3 ubiquitin ligase Ltn1p was recently found to mark the nascent peptides produced from nonstop mRNAs for proteasomal degradation ${ }^{68}$, revealing an interesting conceptual analogy to the bacterial tmRNA-based surveillance system. In bacteria, a unique bi-functional aminoacylated transfer-messenger RNA (tmRNA) binds to the stalled ribosome, allows it to resume translation and serves as a template for addition of a peptide tag to the nascent polypeptide chain that induces its rapid proteolysis ${ }^{73}$. Concerning $k_{\text {normal }} / k_{\text {aberrant }}$ ratios (Figure 2), nonstop mRNAs are presumably efficiently recognized aberrant 
transcripts with the balance tilted strongly towards decay, whereas the frequency and duration of ribosome stalling on NGD substrates to a large extent will depend on extrinsic factors (e.g. temperature, availability of helicases, tRNA concentration, etc.). Thus, although specific examples of gene regulation by NGD have not yet been reported, a rich potential for exploitation of QC factors exists.

\section{NMD at the crossroad between $Q C$ and gene regulation}

NMD represents the most intensively studied QC system, not the least because it has long been recognized as an important modulator of the clinical manifestations of about $30 \%$ of all known disease-associated mutations ${ }^{74}$. NMD has traditionally been portrayed as a QC process that rids cells of faulty mRNAs with prematurely truncated ORFs. However, transcriptome-wide studies from different species have revealed a significant number of functional mRNAs targeted by NMD, demonstrating that it also undertakes translationdependent post-transcriptional gene regulation (reviewed in $27-29,75$ ). Physiological NMD targets are usually downregulated moderately $(2-5$ fold, ${ }^{76}$ ), whereas classical PTC-containing substrates tend to swing the balance towards decay more dramatically by reducing transcript levels $10-50$ fold.

The precise mode of selecting mRNAs for the NMD pathway is not yet understood. However, data from both $S$. cerevisiae and human cells suggest an evolutionarily conserved core mechanism at the heart of which there is a kinetic competition between PABPC1 (the major human cytoplasmic PABP Pab1p in yeast) and the NMD factor UPF1 for a mutually exclusive interaction with eRF3 (Sup35p in yeast) ${ }^{77,78}$ (Figure 5). According to this model, the 
eRF3-PABC1 interaction is required for proper mRNA translation termination, while the competing eRF3-UPF1 interaction represents the first step towards NMD (reviewed in ${ }^{79}, 80$ ). A key determinant for efficient eRF3-PABPC1 interaction is the physical distance between eRF3 bound to the ribosome stalled at the termination codon and PABPC1 bound to the poly $(A)$ tail. The larger this distance, the less efficient is the eRF3-PABPC1 interaction, increasing the likelihood of UPF1 interference and congregation of other NMD components. Consequently, any mRNP rearrangements that alter the structure of the 3' UTR are predicted to affect mRNA half-life and comprise a possibility for gene regulation ${ }^{80,81}$. This mode of regulation has so far only been demonstrated with engineered reporter gene constructs ${ }^{81}$ and physiological examples are awaiting discovery.

After identification as an NMD substrate (i.e. after sorting to the right pan in Figure $2 \mathrm{~A}$ ), the RNA moiety of the mRNP is degraded in different ways depending on the species. In yeast, degradation of NMD substrates involves deadenylation, decapping and exonucleolytic decay ${ }^{82-85}$. In Drosophila and human cells, the metazoan-specific NMD factor and endonuclease SMG6 cuts substrates in the vicinity of the stalled ribosome ${ }^{86-88}$, although there is also evidence for exonucleolytic decay triggered by deadenylation and decapping in human cells ${ }^{89-91,92}$.

Given its role as an effector of $3-10 \%$ of all cellular mRNAs ${ }^{75}$, the activity and substrate selectivity of the NMD system is expected to be controlled by extrinsic signals. For example, during the differentiation of myoblast cells to myotubes, the overall efficiency of NMD diminishes as a consequence of the instigation of another mRNA turnover process called 
Staufen-mediated mRNA decay, which competes for the rate-limiting UPF1 protein. This leads to de-repression of the myogenin mRNA, which encodes a protein required for myogenesis ${ }^{93}$. Similarly, cellular stress such as hypoxia or amino acid deprivation has also been found to reduce NMD potency, resulting in increased levels of numerous mRNAs encoding proteins associated with the respective stress response ${ }^{94,95}$. Furthermore, a recent discovery revealed that inhibition of NMD in neurons of the developing brain up-regulates NMD-sensitive mRNAs encoding neural-specific factors ${ }^{96}$. Interestingly, the underlying mechanism conferring NMD down-modulation depends on binding of the developmentally regulated neuron-specific microRNA miR-128 to the 3'UTR of the UPF1 mRNA, and miR-128 overexpression or UPF1 depletion in neural stem cells individually promoted a neural differentiation phenotype ${ }^{96}$. Finally, two recent publications revealed auto-regulation of NMD factors by demonstrating that most mRNAs encoding these factors are themselves targeted by NMD 76, 97 . While this feedback control on the one hand serves to buffer NMD activity against uncontrolled fluctuations caused by genetic and environmental perturbations, Wilkinson and colleagues also identified cell type-specific and developmental regulation of the NMD pathway ${ }^{97}$. Altogether, these examples emphasize the role of NMD in physiological gene expression regulation and most likely represent only the tip of a slowly surfacing iceberg.

\section{Concluding remarks}

By examples we have aimed to illustrate how the distinction between QC and gene regulation is often blurred. Future discoveries and more detailed 
dissection of the various molecular processes affecting the quality and quantity of expressed genetic information will further scrutinize this concept and reveal its validity and limitation. Irrespectively of the specific model, current data demonstrate that the life of an mRNA is a tightrope walk with decay factors lurking all around. mRNP constituents can be friend or foe and much remains to be learned about when mRNA interactors function as bodyguards or assassins. Studies of mRNP component's 'group dynamics' should reveal some of these secrets.

\section{Acknowledgments}

We thank Manfred Schmid, Hasmik Yepiskoposyan, Søren Lykke-Andersen and Francoise Stutz for valuable comments on the manuscript. Research in the lab of O.M. is supported by the Swiss National Science Foundation (31003A-127614; CRSII3 136222), the European Research Council (StG 207419) and the Kanton Bern. Research in the lab of T.H.J is supported by the Danish National Research, the NOVO Nordisk and the Lundbeck foundations as well as the Danish Cancer Society. 


\section{Figure legend}

Figure 1. Quality control (QC) of mRNP occurs at several checkpoints along the pathway of gene expression. Errors during RNA transcription, capping, splicing, polyadenylation, nuclear export and translation can all lead to faulty transcripts that are retained and/or removed by degradation activities (thin red arrows). The fittest fraction of cellular mRNAs avoid QC to be correctly processed and assembled into functional mRNPs, that engage as templates for protein synthesis before they finally are also degraded (thick blue arrow).

Figure 2. Schematic illustration of the balance between mRNP function and decay. The amount of mRNP persisting after a given step in its biogenesis or after a functional task is determined by the net outcome between two competing rate constants: the rate of mRNP production/function ( $\left.\mathrm{k}_{\text {normal }}\right)$ and the rate of commitment to decay ( $\left.k_{\text {aberrant }}\right)$. Modulating this balance alters mRNP abundance and depending on the perspective, this can be perceived as removal of faulty mRNP $(A)$ or as changing the relative mRNP distribution in the cell (B).

(A) When applied to the population of a single mRNP species (individual mRNPs denoted by a hexagon) that contains a majority of functional particles (green) and a smaller number of defective, or less functional, particles (red), the competition between $\mathrm{k}_{\text {normal }}$ and $\mathrm{k}_{\text {aberrant }}$ identifies and removes aberrant mRNP. Thus, the system performs QC.

(B) When applied to a population of different mRNP species (here three depicted as triangles, squares and ovals, respectively) that rely on the same assembly, processing and degradation activities, extrinsically induced 
changes of one of these parameters will affect $k_{n o r m a l} / k_{a b a r r e n t}$ differently for each individual mRNP species. This will result in an altered relative abundance of the different mRNP species. Thus, the same molecular process as in $(A)$ is now performing gene regulation.

Figure 3. Gene regulation accomplished by the selective recruitment of nuclear QC factors. (A) Auto-regulation of Nrd1p levels in $S$. cerevisiae is achieved by the premature transcription termination of the NRD1 gene directed by the binding of the Nrd1p/Nab3p/Sen1p complex (blue oval) to the nascent NRD1 RNA. Subsequent exosome (red pacman) recruitment results in the elimination of the prematurely terminated transcripts. (B) Nab2p, the nuclear PABP of $S$. cerevisiae, negatively regulates its own expression by binding to a stretch of 26 genomically encoded adenosines (A26) in the 3' UTR of the NAB2 RNA. In the absence of Nab2p at this site (low intracellular Nab2p concentrations), Rrp6p of the nuclear exosome trims the 3' end of the transcript down to the A26 sequence, whereafter poly $(A)$ polymerase (Pap1p) produces a poly $(\mathrm{A})$ tail, leading to stable $N A B 2$ RNA. At high intracellular Nab2 $p$ levels, the factor binds to the A26 sequence and directs complete target degradation by Rrp6p, reducing NAB2 RNA levels. (C) A set of mRNAs coding for meiosis-specific proteins is rapidly degraded in vegetative $S$. pombe cells due to the direct recruitment of Rrp6p and the exosome by the nuclear PABP Pab2p. Exosome/Pab2p presence is furthered, and hence specified, by the RNA-binding protein Mmi1p, which targets the determinant of selective removal (DRS) regions in the 3' UTRs of these mRNAs. During meiosis, Mmi1p is sequestered away from DRS-containing mRNAs into 
meiosis-specific foci, so-called 'Mei2 dots', thereby allowing for the cytoplasmic accumulation of DRS-containing transcripts.

Figure 4. Gene regulation accomplished by modulating the activity of nuclear QC factors. PHO84 gene activity in $S$. cerevisiae is controlled by the overlapping production of asRNA. (A) In young cells, Rrp6p is targeted to PHO84 asRNA resulting in unperturbed transcription of PHO84 RNA. (B) In aged cells, however, Rrp6p recruitment to the PHO84 locus is diminished, allowing asRNA to accumulate with the consequent dampening of PHO84 RNA transcription.

Figure 5. Model for NMD-mediated gene regulation. Metazoan PABP and UPF1 proteins compete for a mutually exclusive interaction with the release factor complex eRF3/eRF1 at the terminating ribosome. Binding of PABP promotes proper translation termination and leaves the mRNA unaffected, whereas binding of UPF1 signals the assembly of an NMD-promoting complex that eventually recruits ribonucleases to trigger rapid mRNA decay. 


\section{References}

1. Moore, M.J. (2005) From birth to death: the complex lives of eukaryotic mRNAs. Science 309, 1514-1518

2. Moore, M.J., and Proudfoot, N.J. (2009) Pre-mRNA processing reaches back to transcription and ahead to translation. Cell 136, 688-700 3. Perales, R., and Bentley, D. (2009) "Cotranscriptionality": the transcription elongation complex as a nexus for nuclear transactions. Mol Cell 36, 178-191

4. Doma, M.K., and Parker, R. (2007) RNA quality control in eukaryotes. Cell 131, 660-668

5. Fasken, M.B., and Corbett, A.H. (2009) Mechanisms of nuclear mRNA quality control. RNA Biol 6, 237-241

6. Houseley, J., and Tollervey, D. (2009) The many pathways of RNA degradation. Cell 136, 763-776

7. Schmid, M., and Jensen, T.H. (2008) The exosome: a multipurpose RNA-decay machine. Trends Biochem Sci 33, 501-510

8. Jimeno-Gonzalez, S., et al. (2010) The yeast 5'-3' exonuclease Rat1p functions during transcription elongation by RNA polymerase II. Mol Cell 37, $580-587$

9. Valen, E., et al. (2011) Biogenic mechanisms and utilization of small RNAs derived from human protein-coding genes. Nat Struct Mol Biol 10. Bousquet-Antonelli, C., et al. (2000) Identification of a regulated pathway for nuclear pre-mRNA turnover. Cell 102, 765-775

11. Burkard, K.T., and Butler, J.S. (2000) A nuclear 3'-5' exonuclease involved in $\mathrm{mRNA}$ degradation interacts with $\operatorname{Poly}(\mathrm{A})$ polymerase and the hnRNA protein Npl3p. Mol Cell Biol 20, 604-616

12. Hilleren, P., et al. (2001) Quality control of mRNA 3'-end processing is linked to the nuclear exosome. Nature 413, 538-542.

13. Libri, D., et al. (2002) Interactions between mRNA export commitment, 3 '-end quality control, and nuclear degradation. Mol Cell Biol 22, 8254-8266

14. Saguez, C., et al. (2008) Nuclear mRNA surveillance in THO/sub2 mutants is triggered by inefficient polyadenylation. Mol Cell 31, 91-103 15. Torchet, C., et al. (2002) Processing of 3'-extended read-through transcripts by the exosome can generate functional mRNAs. Mol Cell 9, 12851296

16. Galy, V., et al. (2004) Nuclear retention of unspliced mRNAs in yeast is mediated by perinuclear Mlp1. Cell 116, 63-73

17. Iglesias, N., et al. (2010) Ubiquitin-mediated mRNP dynamics and surveillance prior to budding yeast mRNA export. Genes Dev 24, 1927-1938

18. Tutucci, E., and Stutz, F. (2011) Keeping mRNPs in check during assembly and nuclear export. Nat Rev Mol Cell Biol 12, 377-384

19. Frischmeyer, P.A., et al. (2002) An mRNA surveillance mechanism that eliminates transcripts lacking termination codons. Science 295, 2258-2261 20. van Hoof, A., et al. (2002) Exosome-mediated recognition and degradation of mRNAs lacking a termination codon. Science 295, 2262-2264 21. Doma, M.K., and Parker, R. (2006) Endonucleolytic cleavage of eukaryotic mRNAs with stalls in translation elongation. Nature 440, 561-564 
22. Losson, R., and Lacroute, F. (1979) Interference of nonsense 76, 5134-5137

23. Thermann, R., et al. (1998) Binary specification of nonsense codons by splicing and cytoplasmic translation. EMBO J 17, 3484-3494

24. Carter, M.S., et al. (1995) A regulatory mechanism that detects premature nonsense codons in T-cell receptor transcripts in vivo is reversed by protein synthesis inhibitors in vitro. J Biol Chem 270, 28995-29003 25. Belgrader, P., et al. (1993) Evidence to implicate translation by ribosomes in the mechanism by which nonsense codons reduce the nuclear level of human triosephosphate isomerase mRNA. Proc Natl Acad Sci U S A $90,482-486$

26. Isken, O., and Maquat, L.E. (2007) Quality control of eukaryotic mRNA: safeguarding cells from abnormal mRNA function. Genes Dev 21, 1833-3856 27. Nicholson, P., et al. (2010) Nonsense-mediated mRNA decay in human cells: mechanistic insights, functions beyond quality control and the double-life of NMD factors. Cell Mol Life Sci 67, 677-700

28. Neu-Yilik, G., and Kulozik, A.E. (2008) NMD: multitasking between mRNA surveillance and modulation of gene expression. Adv Genet 62, 185243

29. Rebbapragada, I., and Lykke-Andersen, J. (2009) Execution of nonsense-mediated mRNA decay: what defines a substrate? Curr Opin Cell Biol 21, 394-402

30. Chang, Y.F., et al. (2007) The Nonsense-Mediated Decay RNA Surveillance Pathway. Annu Rev Biochem 76, 15.11-15.24

31. Nandakumar, J., et al. (2008) RNA repair: an antidote to cytotoxic eukaryal RNA damage. Mol Cell 31, 278-286

32. Saguez, C., et al. (2005) Formation of export-competent mRNP: escaping nuclear destruction. Curr Opin Cell Biol 17, 287-293

33. Schmid, M., and Jensen, T.H. (2010) Nuclear quality control of RNA polymerase II transcripts. WIRES RNA 1, 474-485

34. Wei, W., et al. (2011) Functional consequences of bidirectional promoters. Trends Genet 27, 267-276

35. Jacquier, A. (2009) The complex eukaryotic transcriptome: unexpected pervasive transcription and novel small RNAs. Nat Rev Genet 10, 833-844 36. Kapranov, P., et al. (2007) RNA maps reveal new RNA classes and a possible function for pervasive transcription. Science 316, 1484-1488

37. Neil, H., et al. (2009) Widespread bidirectional promoters are the major source of cryptic transcripts in yeast. Nature 457, 1038-1042

38. Seila, A.C., et al. (2008) Divergent transcription from active promoters. Science 322, 1849-1851

39. Thiebaut, M., et al. (2006) Transcription termination and nuclear degradation of cryptic unstable transcripts: a role for the nrd1-nab3 pathway in genome surveillance. Mol Cell 23, 853-864

40. Core, L.J., et al. (2008) Nascent RNA sequencing reveals widespread pausing and divergent initiation at human promoters. Science 322, 1845-1848 41. Preker, P., et al. (2008) RNA exosome depletion reveals transcription upstream of active human promoters. Science 322, 1851-1854 
42. Preker, P., et al. (2011) PROMoter uPstream Transcripts share characteristics with mRNAs and are produced upstream of all three major types of mammalian promoters. Nucleic Acids Res

43. Lubas, M., et al. (2011) Interaction profiling identifies the human nuclear exosome targeting complex. Mol Cell 43, 624-637

44. David, L., et al. (2006) A high-resolution map of transcription in the yeast genome. Proc Natl Acad Sci U S A 103, 5320-5325

45. Kuehner, J.N., and Brow, D.A. (2008) Regulation of a eukaryotic gene by GTP-dependent start site selection and transcription attenuation. Mol Cell 31, 201-211

46. Martens, J.A., et al. (2005) Regulation of an intergenic transcript controls adjacent gene transcription in Saccharomyces cerevisiae. Genes Dev 19, 2695-2704

47. Thiebaut, M., et al. (2008) Futile cycle of transcription initiation and termination modulates the response to nucleotide shortage in S. cerevisiae. Mol Cell 31, 671-682

48. Arigo, J.T., et al. (2006) Regulation of yeast NRD1 expression by premature transcription termination. Mol Cell 21, 641-651

49. Kim, K.Y., and Levin, D.E. (2011) Mpk1 MAPK association with the Paf1 complex blocks Sen1-mediated premature transcription termination. Cell 144, 745-756

50. Roth, K.M., et al. (2005) The nuclear exosome contributes to autogenous control of NAB2 mRNA levels. Mol Cell Biol 25, 1577-1585 51. Roth, K.M., et al. (2009) Regulation of NAB2 mRNA 3'-end formation requires the core exosome and the Trf4p component of the TRAMP complex. RNA 15, 1045-1058

52. Ciais, D., et al. (2008) The mRNA encoding the yeast ARE-binding protein Cth2 is generated by a novel 3' processing pathway. Nucleic Acids Res 36, 3075-3084

53. Harigaya, Y., et al. (2006) Selective elimination of messenger RNA prevents an incidence of untimely meiosis. Nature 442, 45-50

54. McPheeters, D.S., et al. (2009) A complex gene regulatory mechanism that operates at the nexus of multiple RNA processing decisions. Nat Struct Mol Biol 16, 255-264

55. St-Andre, O., et al. (2010) Negative regulation of meiotic gene expression by the nuclear poly(a)-binding protein in fission yeast. $J$ Biol Chem 285, 27859-27868

56. Yamanaka, S., et al. (2010) Importance of polyadenylation in the selective elimination of meiotic mRNAs in growing $\mathrm{S}$. pombe cells. EMBO J 29, 2173-2181

57. Lemay, J.F., et al. (2010) The nuclear poly(A)-binding protein interacts with the exosome to promote synthesis of noncoding small nucleolar RNAs. Mol Cell 37, 34-45

58. Libri, D. (2010) Nuclear poly(a)-binding proteins and nuclear degradation: take the mRNA and run? Mol Cell 37, 3-5

59. Camblong, J., et al. (2009) Trans-acting antisense RNAs mediate transcriptional gene cosuppression in S. cerevisiae. Genes Dev 23, 15341545 
60. Camblong, J., et al. (2007) Antisense RNA stabilization induces transcriptional gene silencing via histone deacetylation in S. cerevisiae. Cell $131,706-717$

61. Houseley, J., et al. (2008) A ncRNA modulates histone modification and mRNA induction in the yeast GAL gene cluster. Mol Cell 32, 685-695 62. van Dijk, E.L., et al. (2011) XUTs are a class of Xrn1-sensitive antisense regulatory non-coding RNA in yeast. Nature 475, 114-117 63. Zofall, M., et al. (2009) Histone H2A.Z cooperates with RNAi and heterochromatin factors to suppress antisense RNAs. Nature 461, 419-422 64. Berretta, J., et al. (2008) A cryptic unstable transcript mediates transcriptional trans-silencing of the Ty1 retrotransposon in S. cerevisiae. Genes Dev 22, 615-626

65. Lardenois, A., et al. (2011) Execution of the meiotic noncoding RNA expression program and the onset of gametogenesis in yeast require the conserved exosome subunit Rrp6. Proc Natl Acad Sci U S A 108, 1058-1063 66. Cole, S.E., et al. (2009) A convergence of rRNA and mRNA quality control pathways revealed by mechanistic analysis of nonfunctional rRNA decay. Mol Cell 34, 440-450

67. LaRiviere, F.J., et al. (2006) A late-acting quality control process for mature eukaryotic rRNAs. Mol Cell 24, 619-626

68. Bengtson, M.H., and Joazeiro, C.A. (2010) Role of a ribosomeassociated E3 ubiquitin ligase in protein quality control. Nature 467, 470-473 69. Passos, D.O., et al. (2009) Analysis of Dom34 and its function in no-go decay. Mol Biol Cell 20, 3025-3032

70. Kobayashi, K., et al. (2010) Structural basis for mRNA surveillance by archaeal Pelota and GTP-bound EF1alpha complex. Proc Natl Acad Sci U S A 107, 17575-17579

71. Graille, M., et al. (2008) Structure of yeast Dom34 - A protein related to translation termination factor eRF1 and involved in No-Go decay. J. Biol. Chem. 283, 7145-7154

72. Shoemaker, C.J., et al. (2010) Dom34:Hbs1 promotes subunit dissociation and peptidyl-tRNA drop-off to initiate no-go decay. Science 330, 369-372

73. Moore, S.D., and Sauer, R.T. (2007) The tmRNA system for translational surveillance and ribosome rescue. Annu Rev Biochem 76, 101124

74. Frischmeyer, P.A., and Dietz, H.C. (1999) Nonsense-mediated mRNA decay in health and disease. Hum Mol Genet 8, 1893-1900

75. Rehwinkel, J., et al. (2006) Nonsense-mediated mRNA decay: Target genes and functional diversification of effectors. Trends Biochem Sci 31, 639646

76. Yepiskoposyan, H., et al. (2011) Autoregulation of the nonsensemediated mRNA decay pathway in human cells. RNA in press

77. Amrani, N., et al. (2004) A faux 3'-UTR promotes aberrant termination and triggers nonsense-mediated mRNA decay. Nature 432, 112-118 78. Singh, G., et al. (2008) A competition between stimulators and antagonists of Upf complex recruitment governs human nonsense-mediated mRNA decay. PLoS Biol 6, e111

79. Amrani, N., et al. (2006) Early nonsense: mRNA decay solves a translational problem. Nat Rev Mol Cell Biol 7, 415-425 
80. Stalder, L., and Muhlemann, O. (2008) The meaning of nonsense.

Trends Cell Biol 18, 315-321

81. Eberle, A.B., et al. (2008) Posttranscriptional gene regulation by spatial rearrangement of the 3' untranslated region. PLoS Biol 6, e92

82. Cao, D., and Parker, R. (2003) Computational modeling and

experimental analysis of nonsense-mediated decay in yeast. Cell 113, 533-

545

83. Mitchell, P., and Tollervey, D. (2003) An NMD pathway in yeast involving accelerated deadenylation and exosome-mediated 3 '-> 5 ' degradation. Mol. Cell 11, 1405-1413

84. Muhlrad, D., and Parker, R. (1994) Premature translational termination triggers mRNA decapping. Nature 370, 578-581

85. Takahashi, S., et al. (2003) Interaction between Ski7p and Upf1p is required for nonsense-mediated 3'-to-5' mRNA decay in yeast. EMBO $\mathrm{J} 22$, 3951-3959

86. Eberle, A.B., et al. (2009) SMG6 promotes endonucleolytic cleavage of nonsense mRNA in human cells. Nat Struct Mol Biol 16, 49-55

87. Gatfield, D., and Izaurralde, E. (2004) Nonsense-mediated messenger RNA decay is initiated by endonucleolytic cleavage in Drosophila. Nature 429, 575-578

88. Huntzinger, E., et al. (2008) SMG6 is the catalytic endonuclease that cleaves mRNAs containing nonsense codons in metazoan. RNA

89. Couttet, P., and Grange, T. (2004) Premature termination codons enhance mRNA decapping in human cells. Nucleic Acids Res 32, 488-494

90. Lejeune, F., et al. (2003) Nonsense-mediated mRNA decay in mammalian cells involves decapping, deadenylating, and exonucleolytic activities. Mol Cell 12, 675-687

91. Yamashita, A., et al. (2005) Concerted action of poly(A) nucleases and decapping enzyme in mammalian mRNA turnover. Nat Struct Mol Biol 12, 1054-1063

92. Muhlemann, O., and Lykke-Andersen, J. (2010) How and where are nonsense mRNAs degraded in mammalian cells? RNA Biol 7, 28-32

93. Gong, C., et al. (2009) SMD and NMD are competitive pathways that contribute to myogenesis: effects on PAX3 and myogenin mRNAs. Genes Dev 23, 54-66

94. Gardner, L.B. (2008) Hypoxic inhibition of nonsense-mediated RNA decay regulates gene expression and the integrated stress response. Mol Cell Biol 28, 3729-3741

95. Gardner, L.B. (2010) Nonsense-mediated RNA decay regulation by cellular stress: implications for tumorigenesis. Mol Cancer Res 8, 295-308 96. Bruno, I.G., et al. (2011) Identification of a microRNA that activates gene expression by repressing nonsense-mediated RNA decay. Mol Cell 42, 500-510

97. Huang, L., et al. (2011) RNA Homeostasis Governed by Cell TypeSpecific and Branched Feedback Loops Acting on NMD. Mol Cell 43, 950-961 


\section{Transcription}

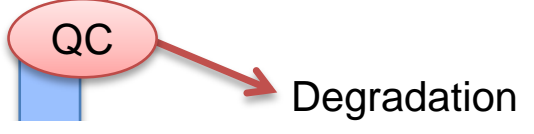

Capping

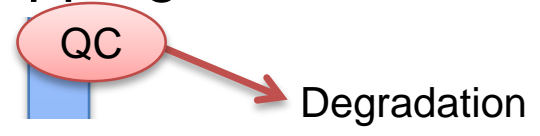

Splicing

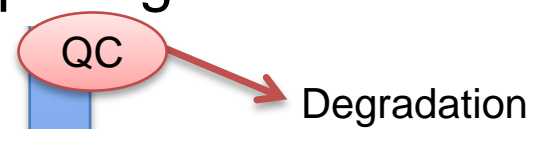

Polyadenylation

QC $\rightarrow$ Degradation

\section{Export to cytoplasm}

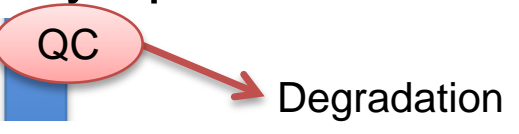

Translation

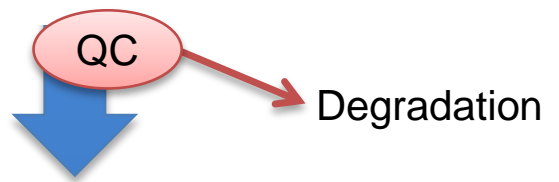

Degradation 


\section{A Removal of faulty mRNP}

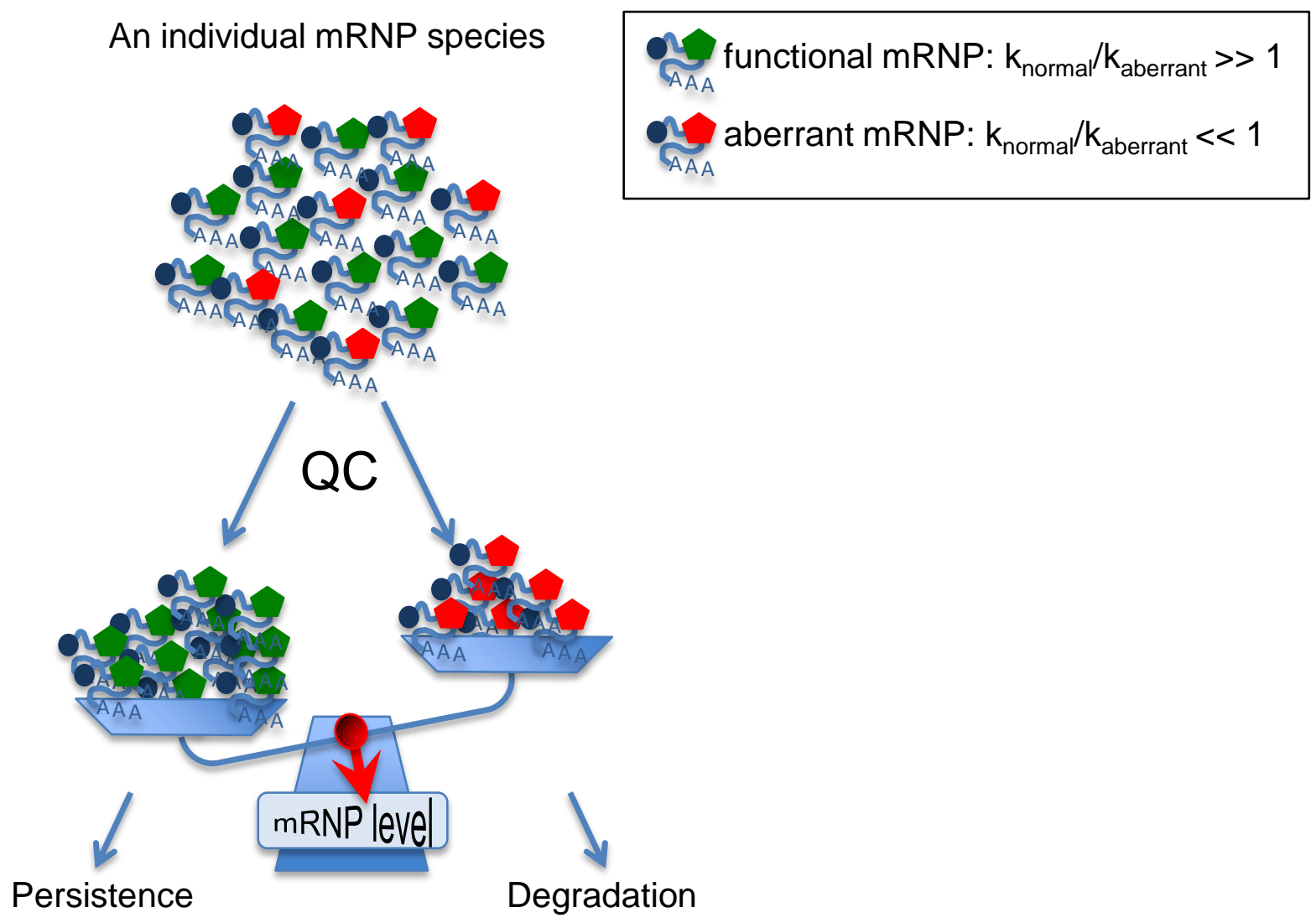


B Change in relative abundance between mRNP species

Population of three individual mRNP species

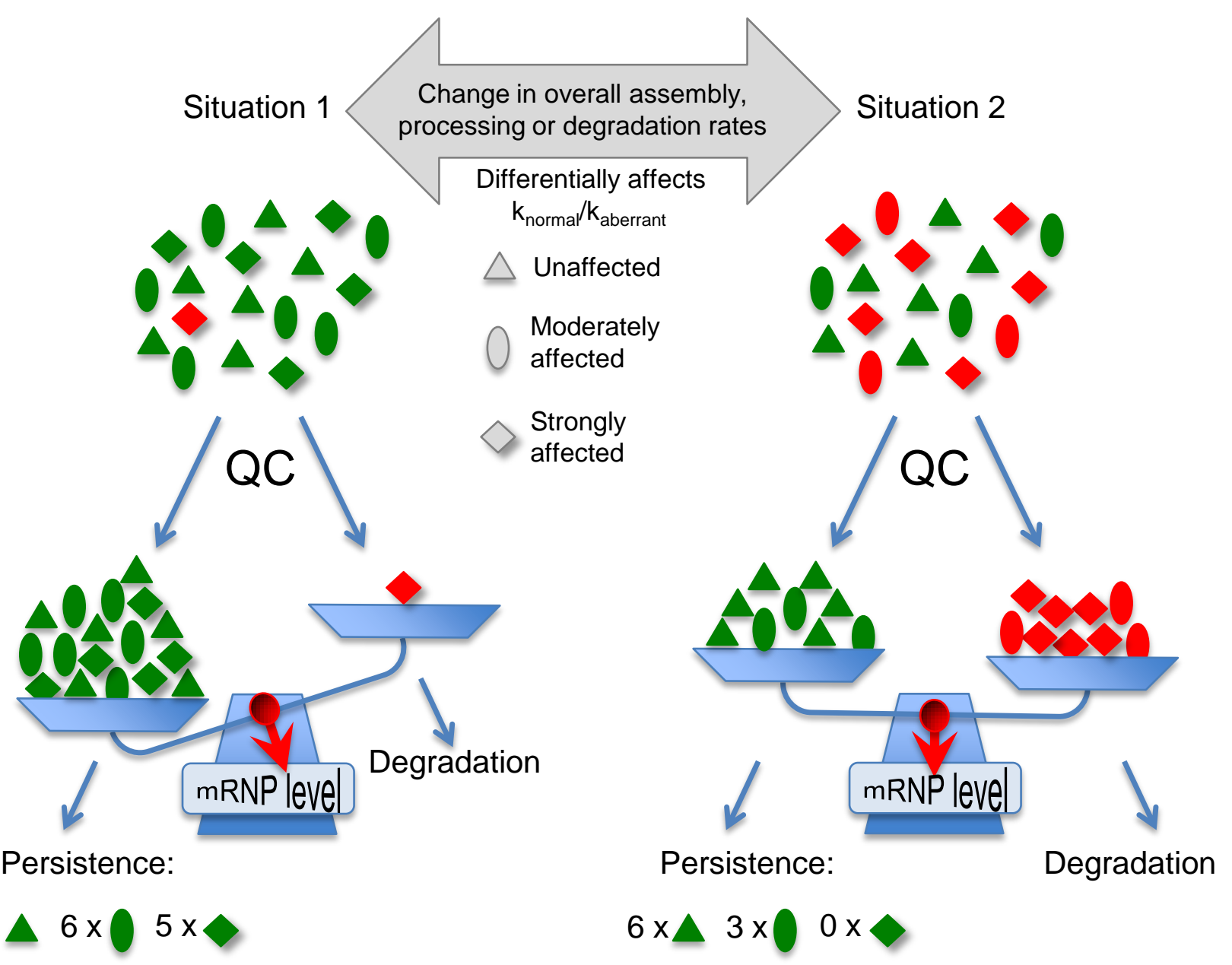




\section{Selective recruitment of $Q C$ factors}

A Nrd1p autoregulation
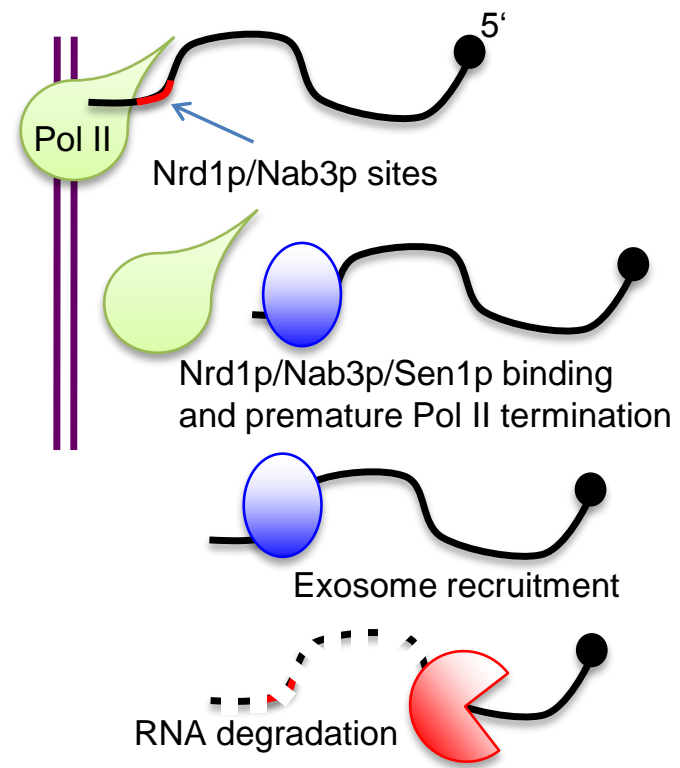

C Control of meiosis-specific mRNAs
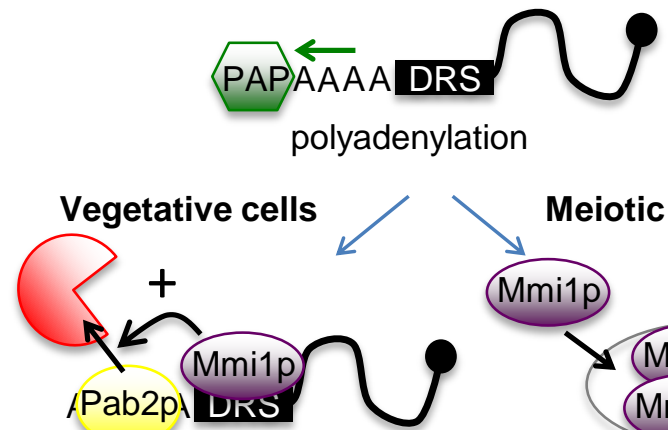

Exosome recruitment

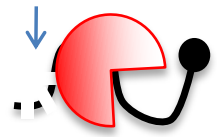

Degradation of DRScontaining mRNA mRNA stable
B

Nab2p autoregulation
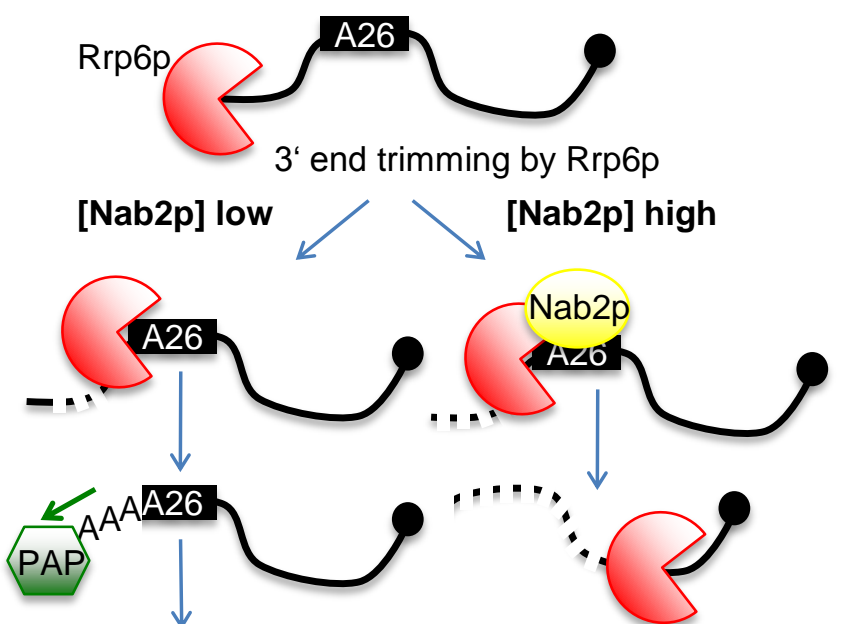

Stable, polyadenylated NAB2 RNA

Rrp6p-mediated degradation of NAB2 RNA

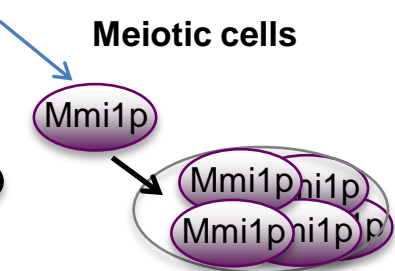

Mmi1p sequestration

AAAAA DRS 


\section{Modulation of QC activity}

\section{PHO84 gene regulation by antisense transcripts}

\section{A Young cells:}

Rrp6p activity high $\rightarrow$ asRNA degradation $\rightarrow$ PHO84 gene activity normal

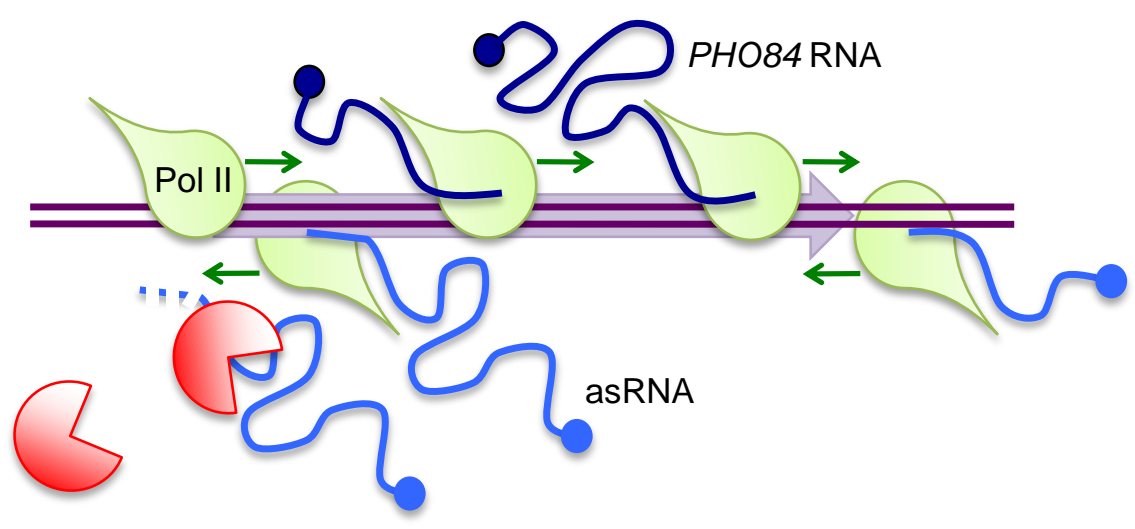

B Aged cells:

Rrp6p activity low $\rightarrow$ asRNAs accumulate $\rightarrow$ PHO84 gene activity decreased

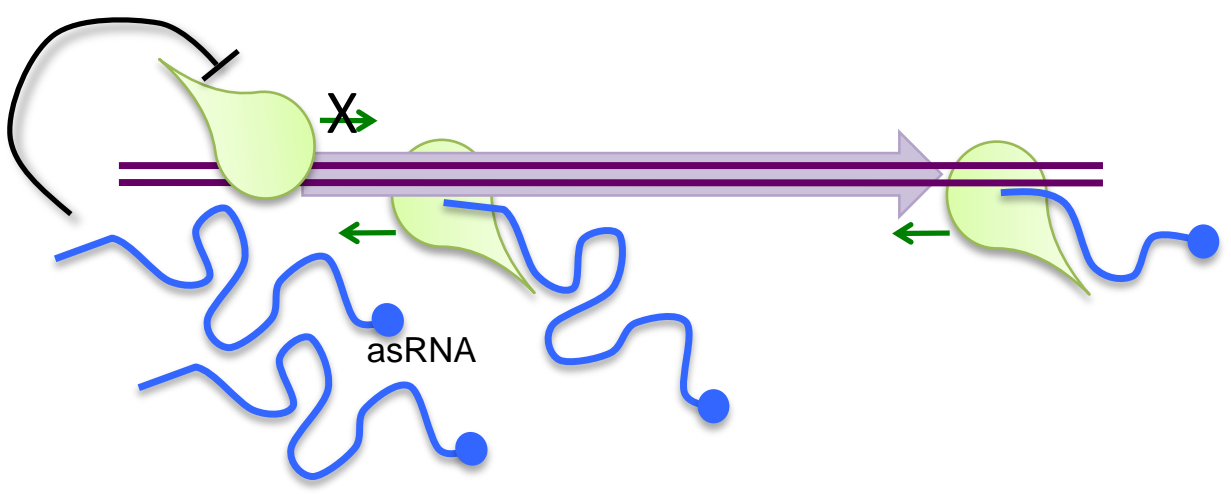




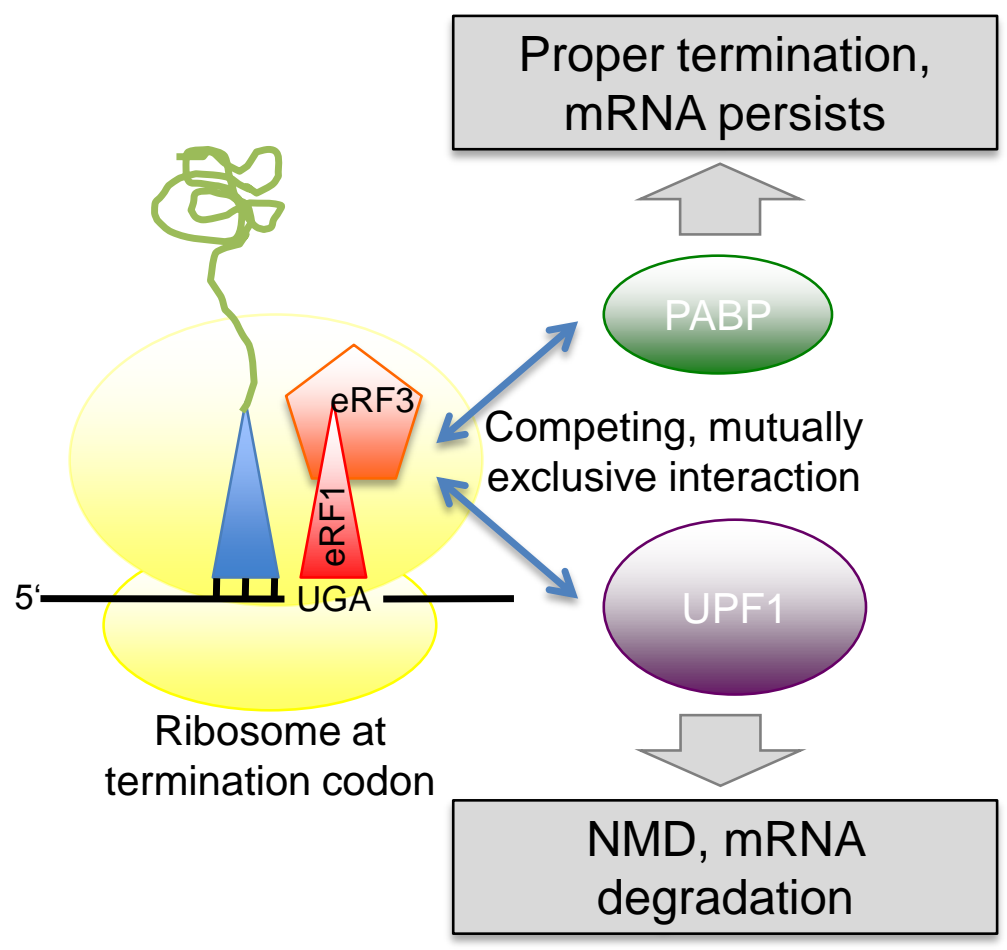

